\title{
Electron ground state $g$ factor in embedded InGaAs quantum dots: An atomistic study
}

\author{
Mustafa Kahraman and Ceyhun Bulutay $\odot^{*}$ \\ Department of Physics, Bilkent University, Ankara 06800, Turkey
}

(Received 15 September 2020; revised 14 January 2021; accepted 19 February 2021; published 3 March 2021)

\begin{abstract}
We present atomistic computations within an empirical pseudopotential framework for the electron $s$-shell ground state $g$ tensor of InGaAs quantum dots (QDs) embedded to host matrices that grant electronic confinement. A large structural set consisting of geometry, size, and molar fraction variations is worked out which also includes a few representative uniform strain cases. The tensor components are observed to display insignificant discrepancies even for the highly anisotropic shapes. The family of $g$-factor curves associated with these parameter combinations coalesces to a single universal one when plotted as a function of the gap energy, thus confirming a recent assertion reached under much restrictive conditions. Our work extends its validity to alloy QDs with various shapes and finite confinement that allows for penetration to the host matrix, placing it on a more realistic basis. Accordingly, the electrons in InGaAs QDs having $s$-shell transition energies close to $1.13 \mathrm{eV}$ will be least susceptible to magnetic field. We also show that low indium concentration offers limited $g$-factor tunability under shape or confinement variations. These findings can be taken into consideration in the fabrication and the use of InGaAs QDs with $g$-near-zero or other targeted $g$ values for spintronic or electron spin resonance-based direct quantum logic applications.
\end{abstract}

DOI: 10.1103/PhysRevB.103.115303

\section{INTRODUCTION}

A single parameter, namely the $g$ factor, succinctly provides a measure of how strongly a charge in an electronic structure couples to an external magnetic field. Compared to its free-electron Dirac equation value of $g_{0}=2$, it can be significantly renormalized in solids, denoted by $g^{*}$, as a result of the spin-orbit interaction [1,2]. Likewise, in semiconductor nanostructures such as quantum dots (QDs), yet another level of renormalization becomes operational by confining the carrier wave function around a heterogeneous region which accordingly tailors the orbital contribution [3], at the same time offering electrical tunability [4-8]. Among these structures, the self-assembled InGaAs QDs particularly stand out where a number of critical quantum technological milestones have been demonstrated, like indistinguishable single-photon sources [9], also on demand [10], spin-resolved resonance fluorescence [11], spin-photon interface [12], entangled photon pairs [13], entanglement swapping [14], as well as simultaneous antibunching and squeezing [15]. Interestingly, the electron spin resonance (ESR) in embedded InGaAs QDs has so far been elusive, with the exception of one report which awaits to be reproduced for more than a decade [16]. As a matter of fact, ESR would be highly welcomed in embedded QDs for the direct magnetic field control of the electron spin over the full Bloch sphere, which was unambiguously showcased much earlier in electrostatically defined gated QDs [17].

An intriguing region that similarly merits attention is where the QD $g$ factor changes sign, which is of relevance to $g$ near-zero QDs $\left(g^{*} \sim 0\right)$. There are a number of reasons why

*bulutay@fen.bilkent.edu.tr this can bring interesting physics. In general the background nuclear spins interact with external magnetic field with a coupling constant about three orders of magnitude smaller than those of free electrons, originating from their Landé factor ratio [18]. Consequently, nuclear magnetic resonance (NMR) and ESR frequencies are off by again a factor of thousand. $g$-near-zero QDs mitigate the ESR and NMR mismatch so that the electron-nucleus counter spin flips become energetically more affordable. This can be utilized to achieve a strong coupling between electron and the nuclear spin bath, similar to the Hartmann-Hahn double resonance [19-21]. From a basic science point of view, $g$-near-zero can promote a spindensity wave state where the spins are oriented perpendicular to the magnetic field [22] and a spin texture of skyrmionic excitations [23]. As to some practical examples, it can facilitate controlled spin rotation by $g^{*}$ tunability provided that it changes sign via electric gating $[4,24]$ or the quantum state transfer between a flying photon qubit and a resident electron spin qubit in a QD [25]. Thus, a deeper understanding of the elements that govern the $g$ factor, especially in InGaAs QDs, is quite valuable for several research directions.

Over the years there has been a number of experimental efforts to better characterize the $g$ factor of InGaAs QDs [26-35]. A complication that arises in most of these magnetoluminescence-based measurements is the extraction from the excitonic $g$ factor that of the electronic contribution $[6,8,27,28,30,31,33,34]$, which hinders its sign, a concern also shared by the magnetocapacitance [29], and photocurrent spectroscopy experiments [35]. Naturally, they need to be supplemented by an electronic structure theory, which has been routinely a variant of the $k \cdot p$ model [36-40], even though more sophisticated alternatives are being developed [41-44]. Another difficulty that virtually affects all experimental 
studies stems from not knowing the precise structural information such as the alloy composition, geometry, and hence the strain profile of the probed single QD. Significant progress was put forward by an inverse approach by feeding in spectroscopical data into theory to find structural models that agree with both the cross-sectional scanning tunneling microscopy and spectroscopy measurements $[45,46]$. However, an ambiguity still remains, as such an approach might overlook the subtleties due to the decaying indium concentration above the QD and the extension of the QD into the wetting layer $[47,48]$. Notwithstanding, as reported by some of these works, this deficient knowledge may not be so critical as it is primarily the gap energy that is directly linked with the $g$ factor [5,30], by that they substantiate the celebrated so-called Roth-Lax-Zwerdling expression which was originally derived for bulk [49]. A recent tight-binding analysis qualitatively supported this by concluding that the dominant contribution to the $g$ factor of nanostructures comes from the bulk term, considering only the compound QDs [50]. Undoubtedly, these assertions merit further theoretical investigation, preferably by an atomistic electronic structure technique that can grant quantitative insights into alloy InGaAs QDs as it is predominantly for the ones studied so far [4,6,7,26-28,30,31].

In this work, we consider $\operatorname{In}_{x} \mathrm{Ga}_{1-x}$ As QDs under a homogeneous compressive hydrostatic strain characteristic of the inner cores of the partially relaxed structures [51-53]. As the embedding material, GaAs is by far the most common choice, but its confinement is rather limited by its bulk band gap of $1.52 \mathrm{eV}$. Other compatible wider gap options are available, such as $\operatorname{In}_{x} \mathrm{Al}_{1-x}$ As with a room temperature energy gap above $2 \mathrm{eV}$ [54], and in the case of $\left(\operatorname{In}_{x} \mathrm{Ga}_{1-x}\right)_{2} \mathrm{O}_{3}$ this reaches $5 \mathrm{eV}$ [55]. Therefore, we assume that each studied QD is embedded into a sufficiently wide band gap matrix that provides confinement for the $s$-shell ground state electron. The QD geometries worked out range from full spherical up to a lens shape as well as various cuts in between with respect to the [111] axis. Independently, the indium fraction is also varied within a uniform alloy profile inside the QD. These structures embody on the order of 10 million atoms including the matrix material which makes it imperative to use an efficient atomistic electronic structure tool. In our case we employ the so-called linear combination of bulk bands (LCBB) which handles such atomic numbers with reasonable computational budget [56]. In the past, we used it in nanocrystals for the linear optical response [57], third-order nonlinear optics [58], electroabsorption [59], and coherent population transfer [60], and in nanowire structures for electronic structure [61] and ballistic transport [62].

Most importantly, among other findings, our work substantiates the conclusion of the aforementioned tight-binding study which reported a universal behavior for $g^{*}$ when plotted with respect to the gap energy [50]. Furthermore, our fitted $g$-factor curve applies to alloy QDs of various shapes with finite confinement that allows for penetration to the matrix as in realistic samples. This result can be beneficial in InGaAs QDs for both achieving a well-controlled ESR as well as avoiding it, depending on the specific purpose. As for the former, if a successful and reproducible ESR is aimed, in the very unfavorable signal-to-noise ratio due to vibrant nuclear spin background [16], there should be no room for ambiguity in the precise frequency of the ESR, hence the $g$ factor. For that matter our fit enables a simple estimate for it based solely on the transition energy, without knowing the precise molar composition and structural information of the QD. Going to the other extreme, if the magnetic effects are desired to be minimized for the $s$-shell ground state electron, as for instance to protect the electron spin qubit [63], then InGaAs QDs with transition energies close to $1.13 \mathrm{eV}$ should be targeted according to our analysis that predicts for them $g^{*} \sim 0$.

The organization of the paper is as follows: In Sec. II we describe the LCBB technique as it is not widely known, together with the $g$-factor expressions. Our computational implementation determines the constraints under which we perform the calculations; in this respect they are crucial and included in Sec. III. Section IV presents our results for a rich variety of QD structures and reveals the underlying universal behavior, followed by our conclusions in Sec. V.

\section{THEORY}

\section{A. LCBB electronic structure technique}

A general necessity in atomistic electronic structure techniques is a large basis set, as in the form of extended plane waves or localized Gaussian orbitals, so as not to compromise accuracy, and this inevitably inflates the computational budget. Yet, when a restricted energy window is of interest, a specialized basis set of modest size selected with physical insight becomes viable, forming the premise of the LCBB method [56]. Here, the basis set is formed by the bulk Bloch functions of the underlying materials within the desired energy range. Hence, the $j$ th stationary state wave function of a nanostructure is approximated by the expansion

$$
\psi_{j}(\mathbf{r})=\frac{1}{\sqrt{N}} \sum_{n, \mathbf{k}, \mu} C_{n \mathbf{k}}^{\mu, j} u_{n \mathbf{k}}^{\mu}(\mathbf{r}) e^{i \mathbf{k} \cdot \mathbf{r}},
$$

where $N$ is the number of primitive unit cells inside the large supercell of the nanostructure, $n$ is the bulk band index, $\mathbf{k}$ is the wave vector within the first Brillouin zone of the underlying lattice, and $\mu$ designates the materials in the set, usually the core and the embedding media. In this expression the cell-periodic part $u_{n \mathbf{k}}^{\mu}(\mathbf{r})$ of the bulk Bloch functions of each material has the Fourier series representation

$$
u_{n \mathbf{k}}^{\mu}(\mathbf{r})=\frac{1}{\sqrt{\Omega_{0}}} \sum_{\mathbf{G}} B_{n \mathbf{k}}^{\mu}(\mathbf{G}) e^{i \mathbf{G} \cdot \mathbf{r}},
$$

where the summation is over the reciprocal lattice vectors $\mathbf{G}$, inside an energy cutoff, and $\Omega_{0}$ is the volume of the primitive cell [2]. The Fourier coefficients $B_{n \mathbf{k}}^{\mu}(\mathbf{G})$ are readily accessible by diagonalizing the bulk Hamiltonian of material $\mu$ at each $\mathbf{k}$ point.

The single-particle Hamiltonian of a nanostructure constitutes the kinetic energy and the crystal potential parts. For the latter we employ the empirical pseudopotentials [64] to describe the atomistic environment

$$
\begin{aligned}
\mathcal{H} & =\mathcal{T}+\mathcal{V}_{\text {xtal }} \\
& =-\frac{\hbar^{2} \nabla^{2}}{2 m_{0}}+\sum_{\mu, \mathbf{R}_{l}, \alpha} W_{\alpha}^{\mu}\left(\mathbf{R}_{l}\right) v_{\alpha}^{\mu}\left(\mathbf{r}-\mathbf{R}_{l}-\mathbf{d}_{\alpha}^{\mu}\right),
\end{aligned}
$$


where $m_{0}$ is the free electron mass, the direct lattice vector $\mathbf{R}_{l}$ indicates the origin for each primitive cell $l$, and $\mathbf{d}_{\alpha}^{\mu}$ specifies the relative coordinate of the basis atom $\alpha$ within the primitive cell. The weight function $W_{\alpha}^{\mu}\left(\mathbf{R}_{l}\right)$ keeps the information about the atomistic composition of the nanostructure by taking values 0 or 1 depending on the type of the atom located at the position $\mathbf{R}_{l}+\mathbf{d}_{\alpha}^{\mu} \cdot v_{\alpha}^{\mu}$ is the local screened spherical atomic pseudopotential of atom $\alpha$ of the material $\mu$ [64].

Hamiltonian matrix elements are evaluated with respect to the LCBB basis states $\{|n \mathbf{k} \mu\rangle\}$ which can be cast into a generalized eigenvalue problem

$$
\sum_{n, \mathbf{k}, \mu}\left\langle n^{\prime} \mathbf{k}^{\prime} \mu^{\prime}\left|\mathcal{T}+\mathcal{V}_{\text {xtal }}\right| n \mathbf{k} \mu\right\rangle C_{n \mathbf{k}}^{\mu, j}=E_{j} \sum_{n, \mathbf{k}, \mu} C_{n \mathbf{k}}^{\mu, j}\left\langle n^{\prime} \mathbf{k}^{\prime} \mu^{\prime} \mid n \mathbf{k} \mu\right\rangle,
$$

which yields the energy $E_{j}$ and the expansion coefficients $C_{n \mathbf{k}}^{\mu, j}$. The explicit forms of these matrix elements are

$$
\begin{aligned}
\left\langle n^{\prime} \mathbf{k}^{\prime} \mu^{\prime} \mid n \mathbf{k} \mu\right\rangle= & \delta_{\mathbf{k}, \mathbf{k}^{\prime}} \sum_{\mathbf{G}}\left[B_{n^{\prime} \mathbf{k}}^{\mu^{\prime}}(\mathbf{G})\right]^{*} B_{n \mathbf{k}}^{\mu}(\mathbf{G}) \\
\left\langle n^{\prime} \mathbf{k}^{\prime} \mu^{\prime}|\mathcal{T}| n \mathbf{k} \mu\right\rangle= & \delta_{\mathbf{k}, \mathbf{k}^{\prime}} \sum_{\mathbf{G}} \frac{\hbar^{2}|\mathbf{k}+\mathbf{G}|^{2}}{2 m_{0}}\left[B_{n^{\prime} \mathbf{k}}^{\mu^{\prime}}(\mathbf{G})\right]^{*} B_{n \mathbf{k}}^{\mu}(\mathbf{G}) \\
\left\langle n^{\prime} \mathbf{k}^{\prime} \mu^{\prime}\left|\mathcal{V}_{\mathbf{x t a l}}\right| n \mathbf{k} \mu\right\rangle= & \sum_{\mathbf{G}, \mathbf{G}^{\prime}}\left[B_{n^{\prime} \mathbf{k}^{\prime}}^{\mu^{\prime}}\left(\mathbf{G}^{\prime}\right)\right]^{*} B_{n \mathbf{k}}^{\mu}(\mathbf{G}) \\
& \times \sum_{\mu^{\prime \prime}, \alpha} \mathcal{V}_{\alpha}^{\mu^{\prime \prime}}\left(\left|\mathbf{k}+\mathbf{G}-\mathbf{k}^{\prime}-\mathbf{G}^{\prime}\right|\right) \\
& \times \mathcal{W}_{\alpha}^{\mu^{\prime \prime}}\left(\mathbf{k}-\mathbf{k}^{\prime}\right) e^{-i\left(\mathbf{k}+\mathbf{G}-\mathbf{k}^{\prime}-\mathbf{G}^{\prime}\right) \cdot \mathbf{d}_{\alpha}^{\mu^{\prime \prime}}}
\end{aligned}
$$

Here, $\mathcal{V}_{\alpha}^{\mu^{\prime \prime}}$ and $\mathcal{W}_{\alpha}^{\mu^{\prime \prime}}$ are the Fourier transforms of atomic pseudopotentials and the weight functions

$$
\begin{gathered}
\mathcal{V}_{\alpha}^{\mu^{\prime \prime}}\left(\left|\mathbf{k}+\mathbf{G}-\mathbf{k}^{\prime}-\mathbf{G}^{\prime}\right|\right)=\frac{1}{\Omega_{0}} \int v_{\alpha}^{\mu^{\prime \prime}}(\mathbf{r}) e^{i\left(\mathbf{k}+\mathbf{G}-\mathbf{k}^{\prime}-\mathbf{G}^{\prime}\right) \cdot \mathbf{r}} d^{3} r \\
\mathcal{W}_{\alpha}^{\mu}\left(\mathbf{k}-\mathbf{k}^{\prime}\right)=\sum_{j} W_{\alpha}^{\mu^{\prime \prime}}\left(\mathbf{R}_{j}\right) e^{i\left(\mathbf{k}-\mathbf{k}^{\prime}\right) \cdot \mathbf{R}_{j}}
\end{gathered}
$$

\section{B. Spin-orbit interaction}

So far, only the spin independent part of the Hamiltonian is considered. Following Hybertsen and Louie [65], the spinorbit interaction can be incorporated as

$$
\mathcal{H}_{\mathrm{SO}}=\sum_{\ell=1}^{\infty}|\ell\rangle V_{\ell}^{\mathrm{SO}}(r) \ell \cdot \sigma\langle\ell|,
$$

where $\ell$ is the orbital angular momentum label, $\sigma$ is the vector Pauli spin operator, and $V_{\ell}^{\mathrm{SO}}(r)$ is the angular-momentumdependent (i.e., nonlocal) radial spin-orbit potential. To simplify, we restrict to the dominant $\ell=1$, i.e., $p$ component, and the spin-orbit matrix elements become

$$
\begin{aligned}
& \left\langle s, \mathbf{K}\left|\mathcal{H}_{\mathrm{SO}}\right| s^{\prime}, \mathbf{K}^{\prime}\right\rangle \\
& \quad=-i\left\langle s|\sigma| s^{\prime}\right\rangle \cdot\left[12 \pi \frac{\mathbf{K} \times \mathbf{K}^{\prime}}{K K^{\prime}} V_{\ell=1}^{\mathrm{SO}}\left(K, K^{\prime}\right)\right] S\left(\mathbf{K}^{\prime}-\mathbf{K}\right),
\end{aligned}
$$

where $\mathbf{K}=\mathbf{k}+\mathbf{G}, \mathbf{K}^{\prime}=\mathbf{k}+\mathbf{G}^{\prime},|s\rangle$ denotes a spinor state, $S\left(\mathbf{K}^{\prime}-\mathbf{K}\right)$ is the bulk static structure factor. $V_{\ell}^{\mathrm{SO}}\left(K, K^{\prime}\right)$ is given by the integral

$$
V_{\ell}^{\mathrm{SO}}\left(K, K^{\prime}\right)=\int_{0}^{\infty} \frac{d r}{\Omega_{0}} r^{2} j_{\ell}(K r) V_{\ell}^{\mathrm{SO}}(r) j_{\ell}\left(K^{\prime} r\right),
$$

with $j_{\ell}$ being the spherical Bessel function of the first kind, and $V_{\ell}^{\mathrm{SO}}(r)$ is chosen as a Gaussian function [66] with a width of 2.25 Bohr radius and its amplitude being a fit parameter, $\lambda_{S}$ as described below. $V_{\ell}^{\mathrm{SO}}\left(K, K^{\prime}\right)$ is computed once and invoked from a look-up table.

\section{C. $g$ factor}

Unlike a free electron, a charge in a nanostructure experiences an anisotropic coupling to an external magnetic field B so that its $g$ factor becomes a rank-2 tensor $\stackrel{\leftrightarrow}{g}$, which in the most general case is characterized by nine linearly independent components [36]. It is described through the Zeeman Hamiltonian

$$
\mathcal{H}_{Z}=\frac{1}{2} \mu_{B} \sigma \cdot \stackrel{\leftrightarrow}{g} \cdot \mathbf{B},
$$

where $\mu_{B}$ is the Bohr magneton. The celebrated $\stackrel{\leftrightarrow}{g}$ expression follows from a spinless electronic structure calculation when the spin-orbit interaction is included as a first-order perturbation [2,67]. This bulk formulation can be extended to QDs in terms of the matrix elements between two confined states $n$ and $j$ as

$$
\begin{aligned}
& \mathbf{p}_{n j}=\frac{(2 \pi)^{3}}{\Omega_{\mathrm{SC}}} \int_{\mathrm{SC}} \psi_{n}^{*}(\mathbf{r}) \mathbf{p} \psi_{j}(\mathbf{r}) d^{3} r, \\
& \mathbf{h}_{n j}=\frac{(2 \pi)^{3}}{\Omega_{\mathrm{SC}}} \int_{\mathrm{SC}} \psi_{n}^{*}(\mathbf{r}) \mathbf{h} \psi_{j}(\mathbf{r}) d^{3} r,
\end{aligned}
$$

where the integrals are over the supercell volume $\Omega_{\mathrm{SC}}, \mathbf{p}$ is the momentum operator, and $\mathbf{h}$ is the spin-orbit related operator defined through $\mathcal{H}_{\mathrm{SO}}=\mathbf{h} \cdot \boldsymbol{\sigma}$; see Eq. (4).

For a chosen state $n$, this yields the $g$ factor

$$
\begin{aligned}
\stackrel{\leftrightarrow}{g}_{n}= & 2 \stackrel{\leftrightarrow}{I}+\frac{2}{i \hbar^{2} m_{0}} \sum_{j l}^{\prime} \frac{1}{\omega_{n j}} \\
& \times\left[\frac{\left(\mathbf{h}_{j l}-\mathbf{h}_{l j}\right)\left(\mathbf{p}_{n j} \times \mathbf{p}_{l n}\right)}{\omega_{j l}}+\frac{\left(\mathbf{h}_{l n}-\mathbf{h}_{n l}\right)\left(\mathbf{p}_{n j} \times \mathbf{p}_{j l}\right)}{\omega_{n l}}\right]
\end{aligned}
$$

where $\stackrel{\leftrightarrow}{I}$ is the $3 \times 3$ identity matrix, the prime over the summation stands for $j \neq l$, and $\omega_{n j}=\left(E_{n}-E_{j}\right) / \hbar$, etc. With some manipulations, it can be shown to be equivalent to the Roth's bulk expression [67]

$$
\begin{aligned}
\stackrel{\leftrightarrow}{g}_{n}= & 2 \stackrel{\leftrightarrow}{I}+\frac{2}{i \hbar^{2} m_{0}} \sum_{j l}^{\prime} \frac{1}{\omega_{n j} \omega_{n l}} \\
& \times\left(\mathbf{h}_{n j} \mathbf{p}_{j l} \times \mathbf{p}_{l n}+\mathbf{h}_{j l} \mathbf{p}_{n j} \times \mathbf{p}_{l n}+\mathbf{h}_{l n} \mathbf{p}_{n j} \times \mathbf{p}_{j l}\right),
\end{aligned}
$$

where in contrast to bulk, here the matrix elements are worked out using nanostructure states as given by Eqs. (8) and (9). 


\section{COMPUTATIONAL IMPLEMENTATION}

In this section we would like to give some important details about our computational model. Foremost, we utilize our recently fitted empirical pseudopotentials for InAs and GaAs under various strain conditions to hybrid density functional theory band structures [68]. In anticipation to reduce matrix sizes, the fit was achieved with about 120 reciprocal lattice vectors within the energy cutoff. For the current work involving QDs having the $\operatorname{In}_{x} \mathrm{Ga}_{1-x}$ As alloy core, we use Vegard's law in mixing the compound InAs and GaAs pseudopotentials. As mentioned above, the spin-orbit interaction over the $p$ states brings a further symmetric spin-orbit coupling parameter $\lambda_{S}$ fitted to experimental spin-orbit splittings for bulk InAs and GaAs [68].

The $\stackrel{\leftrightarrow}{g}_{n}$ expression in Eq. (11) requires, in principle, all of the QD states but especially those energetically close to the state $n$ under investigation. This demands well characterization of a large number of electronic states which hinges upon the strength of the LCBB basis set. Recall that in our formulation the spin-orbit interaction enters as a perturbation, hence the wave functions are spinless. We employ the bulk bands of the spinless top four (four) valence and the lowest four (one) conduction bands of the strained core (matrix) material. For either case, basis sets are formed from a three-dimensional $5 \times 5 \times 5$ grid in the reciprocal space centered around the $\Gamma$ point. Its convergence was checked for the supercell size we adopted for calculations in this work. The final LCBB basis sets typically contain some two thousand elements.

The non-self-consistent nature of the empirical pseudopotentials [64] entails an additional bulk parameter to have a desirable band alignment under strain. Following Williamson et al., this is implemented as a hydrostatic strain-dependent pseudopotential formed as

$$
V(q ; \epsilon)=\left[1+\gamma \epsilon_{H}\right] V(q),
$$

where $\gamma$ is the accompanying fitting parameter and $\epsilon_{H}=$ $\epsilon_{x x}+\epsilon_{y y}+\epsilon_{z z}$ refers to hydrostatic strain [66]. We should note that in all of the calculations, we assume a uniformly strained QD so that the same lattice constant applies over the full supercell. This greatly simplifies the computational tasks like the choice of the basis sets and allows the use of the standard fast Fourier transform (FFT) in Eq. (3) [69]. Even then, representing the existing theoretical and experimental band offset data [70-73] becomes quite challenging mainly due to strain-related band gap as well as lineup variations for all the structures worked out in this study. This necessitates the use of several different artificial matrices, in each case lattice matched to core (strained) QD and with band gaps ranging from $1.52 \mathrm{eV}$ (GaAs) to $5 \mathrm{eV}$ [such as $\left(\mathrm{In}_{x} \mathrm{Ga}_{1-x}\right)_{2} \mathrm{O}_{3}$ ]. The conduction band offset values depending on indium mole fraction and strain range between $65 \mathrm{meV}$ to $245 \mathrm{meV}$ to ensure the confinement $s$-shell ground state electron.

In regard to the above simplifications of our computational model, the pioneering works expressed that the embedded InGaAs QD geometry and the structural relaxation result in a position-dependent strain within the QD, see, e.g., Refs. [51,66,71]. A number of $k \cdot p$ and tight-binding studies elaborated on their electronic properties using highly sophisticated QD structural information, see, e.g., Refs. [41,74,75].

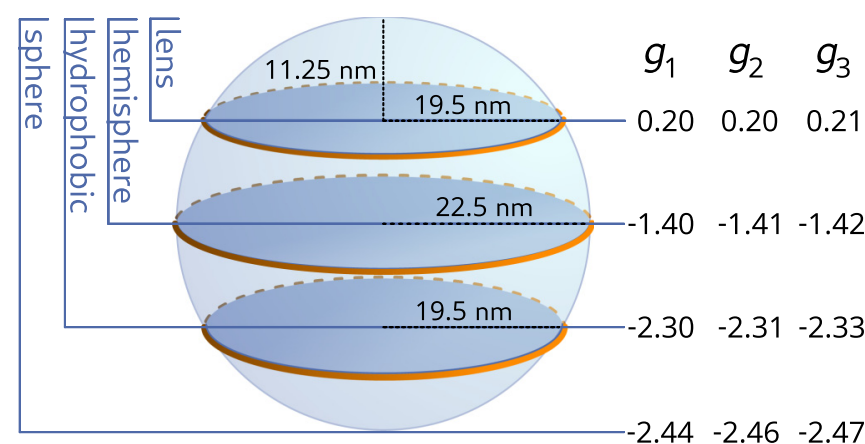

FIG. 1. The three principal $\stackrel{\leftrightarrow}{g}$ values of embedded InAs QDs under $2 \%$ homogeneous compressive strain. The four geometries originate from a sphere by cutting with a (111) plane producing lens, hemisphere, and hydrophobic-contact-angle spherical domes.

On the other hand, from the LCBB point of view inhomogeneous strain would call for nonuniform FFT which slows down the calculations drastically, even taking into account recently developed packages [76,77]. Moreover, a much richer strained basis set is required that compounds the computational overhead. For this reason, we opt for a few uniform cases, representative of average strain present in typical QDs [51-53]. This leaves outside the effect of inhomogeneous strain on the $g$ factor, thereby pinpointing a direction along which our approach can be further improved.

\section{RESULTS}

\section{A. Cuts from a sphere}

We start with the compound InAs spherical QD of $45 \mathrm{~nm}$ diameter embedded in a host matrix, where the QD is under a $2 \%$ compressive strain, corresponding to a hydrostatic strain of $\epsilon_{H}=-0.06$. In Fig. 1 we see how the principal values of $\stackrel{\leftrightarrow}{g}$ vary when the sphere is successively cut by a (111) oriented plane, producing in addition to a sphere a hydrophobic-contact-angle-, hemispherical-, and lens-shaped QD. As expected, the increasing confinement gradually modifies $g^{*}$ from -2.47 to 0.21 so that $g^{*} \sim 0$ would be attained for a lens shaped QD with a bigger diameter than the one in Fig. 1. The three principal values of $\stackrel{\leftrightarrow}{g}$ marginally deviate from each other even though they become exceedingly of anisotropic shapes toward the lens QD. The largest difference is about 0.03 that occurs for the spherical QD which indicates the numerical accuracy of our calculations. This lack of anisotropy in $\stackrel{\leftrightarrow}{g}$ is ubiquitous for all the structures studied in this work. Therefore, we shall display its major principal component in the plots to follow.

Next, choosing the spherical, hemispherical, and lens geometries from Fig. 1, we consider how both $g$ factor and the highest occupied molecular orbital (HOMO) to lowest unoccupied molecular orbital (LUMO) energy gap evolve with the indium molar fraction for alloy $\operatorname{In}_{x} \mathrm{Ga}_{1-x}$ As QDs. Figure 2 illustrates the family of curves belonging to each shape with a diameter around $46 \mathrm{~nm}$, and for the lens ones of height about 11-12 $\mathrm{nm}$ for the QD strain value of $-2 \%$, i.e., $\epsilon_{x x}=$ $\epsilon_{y y}=\epsilon_{z z}=-0.02$. In Fig. 2(a) the geometric sensitivity in $g^{*}$ reveals itself toward the indium-rich composition, where 

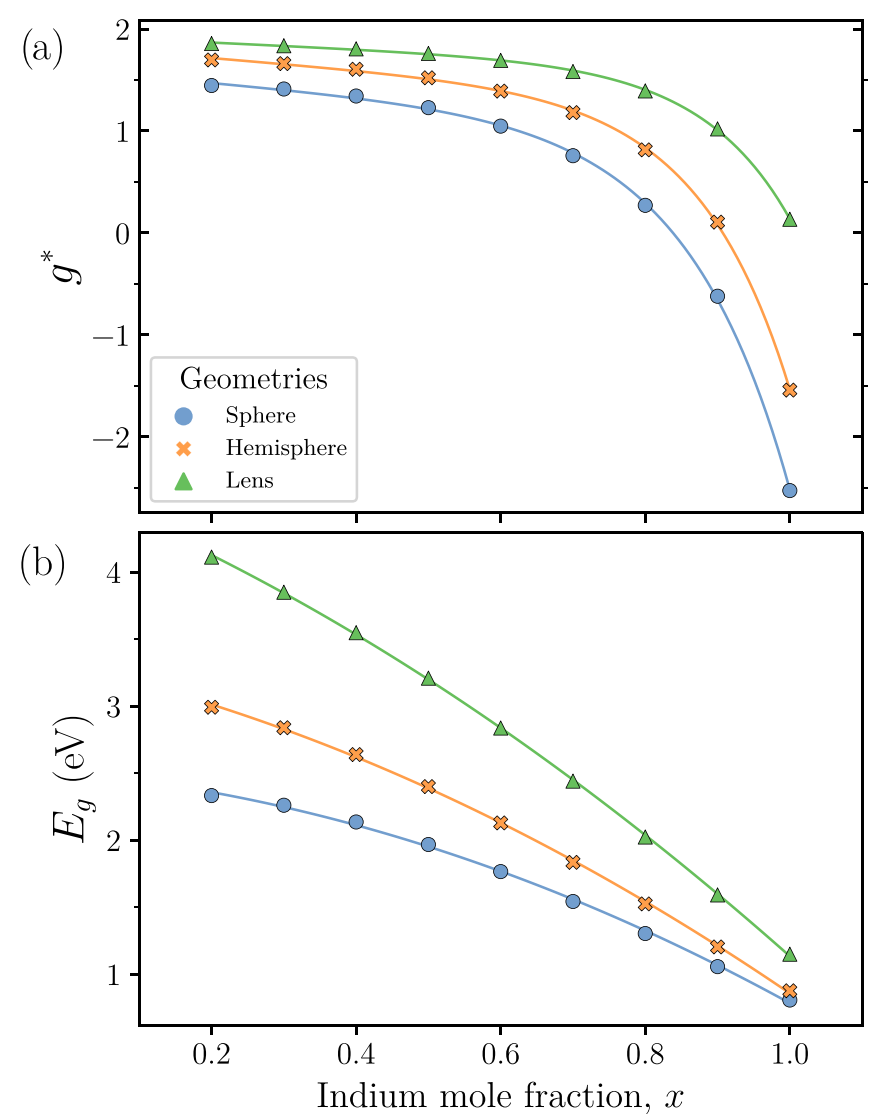

FIG. 2. Variation of (a) $g$ factor and (b) HOMO-LUMO energy gap, $E_{g}$ of the spherical-, hemispherical-, and lens-shaped $\mathrm{In}_{x} \mathrm{Ga}_{1-x}$ As QDs under $-2 \%$ homogeneous strain as a function of the indium molar fraction. All of them have the same diameter of $46 \mathrm{~nm}$, and the lens QDs have a height about 11-12 nm. Solid lines are to guide the eye.

the sign change also takes effect. In that respect, indium-poor QDs offer very limited $g$ tunability. The accompanying energy gaps in Fig. 2(b) merit some explanation as one would expect an opposite trend based on the quantum size effect consideration which would decrease as the indium content is reduced toward GaAs because of the heavier effective mass of GaAs. This tendency is more than compensated by the increase in the gap energy due to increasing gallium fraction. It is considerably boosted under strain as the bulk band gap deformation potential of $\mathrm{GaAs}(-8.69 \mathrm{eV})$ is about $50 \%$ larger than InAs $(-5.95 \mathrm{eV})$ [68]. This results in an overall increase in the HOMO-LUMO energy seen in Fig. 2(b) as the indium content is lowered toward GaAs. The fact that the structures are embedded into wider gap matrices allows us to keep track of the full variation in the $g$ factor without losing confinement up to energies as high as $4 \mathrm{eV}$. The opposite behavior of $g^{*}$ and $E_{g}$ will be a recurring theme also in the following results.

\section{B. Dimensional dependence in lens QDs}

In the remaining sections we concentrate on the lens QDs as being the prevalent embedded self-assembled QD shape. First, we present in Fig. 3 the set of curves for a wide range of indium mole fractions, and for two different strains, all at

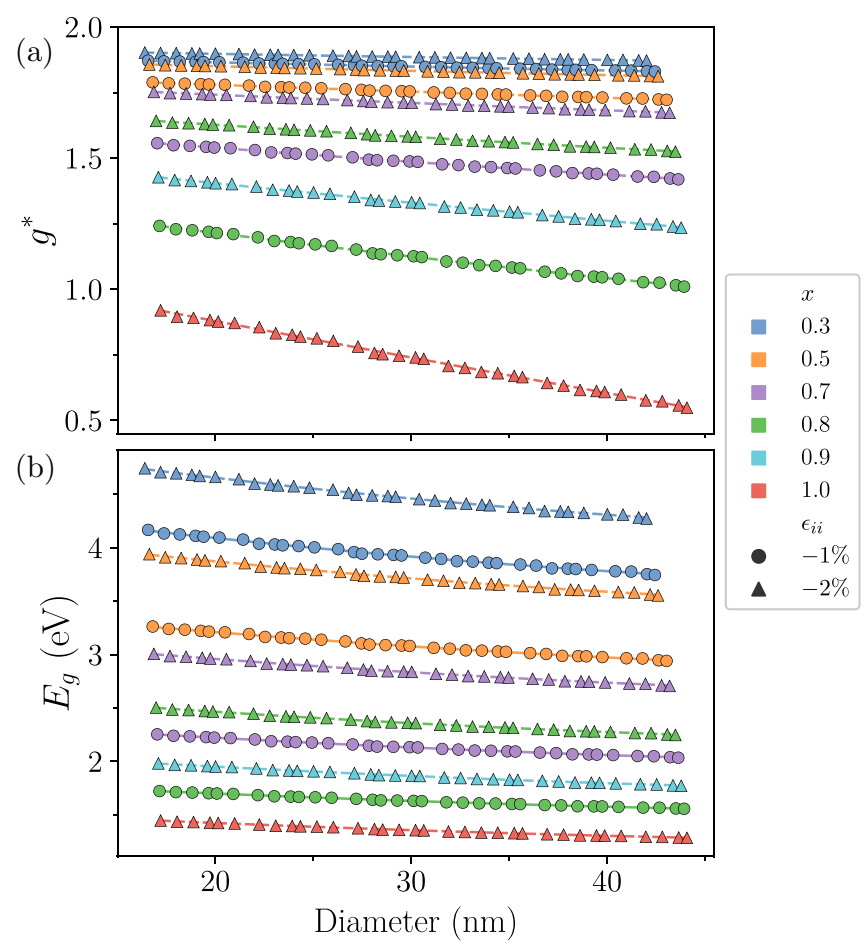

FIG. 3. Variation of (a) $g$ factor and (b) HOMO-LUMO energy gap $E_{g}$ versus the diameter of the lens QD. The family of curves are all at a fixed aspect ratio of $h / D=0.2$ for different indium mole fraction and strain values. Dashed lines are to guide the eye.

a fixed aspect ratio (height over diameter), $h / D=0.2$. Most notably, in Fig. 3(a) compressive strain raises the $g$ factor. The reason is predominantly the widening in HOMO-LUMO energy gap with compressive strain as shown in Fig. 3(b), due to negative band gap deformation potential of both InAs and GaAs [68]. Its connection with the $g$ factor is directly visible from the energy denominators in Eqs. (10) and (11), where their increase causes reduced renormalization with respect to the free-electron value. This is reminiscent of the $k \cdot p$ conduction band effective mass expression, where $m^{*}$ decreases as the band gap increases [78]. A further remark is that, as in Fig. 2, for low indium concentration, $g^{*}$ approaches freeelectron value and becomes largely QD diameter independent. As the indium content increases, so does the contribution of spin-orbit interaction, which together with decreasing energy gap both lower $g^{*}$ and instate its size dependence.

Another set of curves follows, this time varying the QD height, keeping the lens basal diameter fixed at $35 \mathrm{~nm}$ as shown in Fig. 4. The general trends are similar, as the increase in $g^{*}$ under compressive strain in Fig. 4(a) can be linked to that of the HOMO-LUMO energy gap in Fig. 4(b). In comparison to Fig. 3, there is a wider change under height and in turn aspect ratio. For the considered lens diameter, $g^{*} \sim 0$ ensues very close to InAs composition. An intriguing observation is that different mole fraction and strain curves can perfectly overlap as in $\left(x=0.5, \epsilon_{i i}=-0.01\right)$ and $(x=$ $\left.0.8, \epsilon_{i i}=-0.03\right)$. This suggests that, as far as $g$ factor is concerned, there can be a universal dependence under a decisive parameter, as we discuss next. 


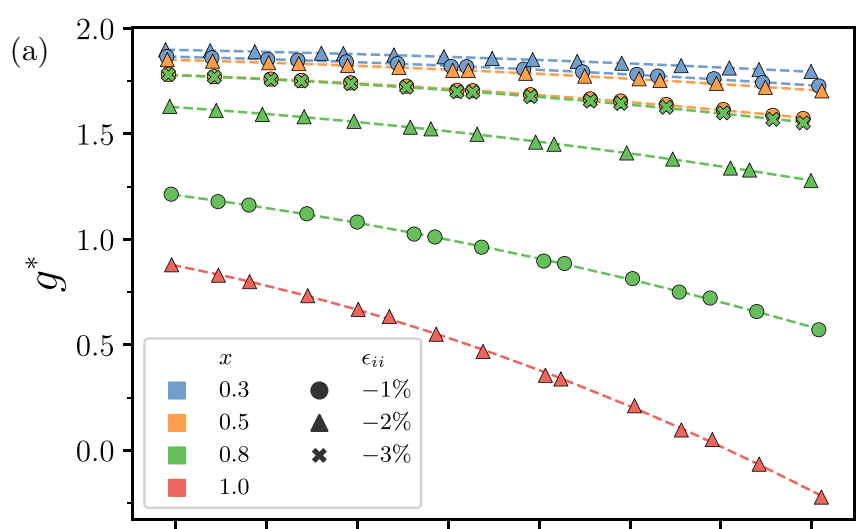

(b)

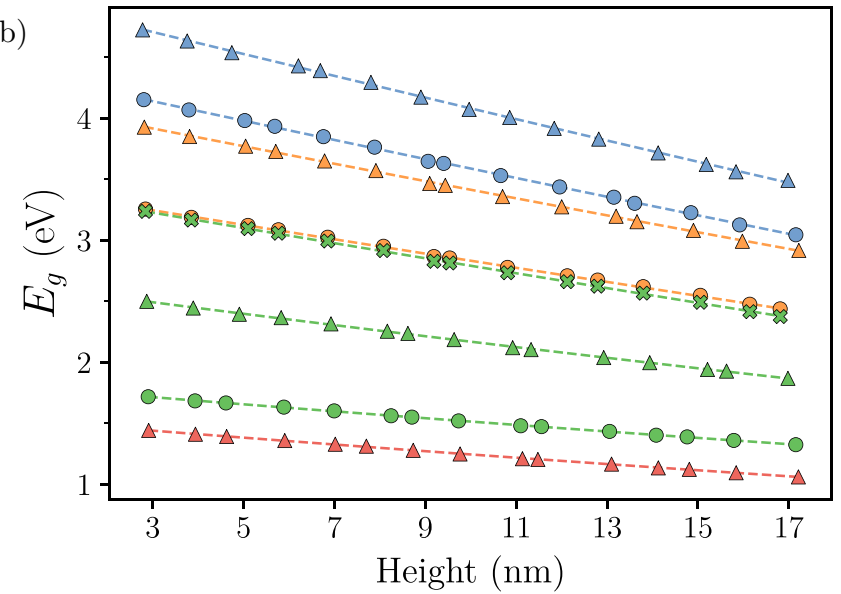

FIG. 4. Variation of (a) $g$ factor and (b) HOMO-LUMO energy gap $E_{g}$ versus the height of the lens QD for a fixed basal diameter of $35 \mathrm{~nm}$. The family of curves are for different indium mole fraction and strain values. Dashed lines are to guide the eye.

\section{Universality with respect to gap energy}

We now recast all the various QD $g$-factor data above as a function of the HOMO-LUMO energy gap $E_{g}$ for each case. In connection to low-temperature magnetoluminescence experiments it can be easily extended to include the excitonic binding energy [59]. When we replot the data in Figs. 2, 3, and 4 in this manner, we observe that all the family of curves for distinct mole fractions, strains, aspect ratios, and heights coalesces to a universal curve as presented in Figs. 5(a), 5(b) and 5(c), respectively. This not only supports the earlier finding of Ref. [50] but also extends it to diverse geometries and alloys while allowing for penetration into surrounding matrix material. All these data can be well represented by a simple curve of the same bulk form [37,49]

$$
g^{*}\left(E_{g}\right)=2-\frac{2.06}{E_{g}\left(E_{g}-0.22\right)},
$$

where $E_{g}$ is in eV. According to Eq. (13), we can predict that the electrons in InGaAs QDs possessing $s$-shell transition energies close to $1.13 \mathrm{eV}$ will be least susceptible to magnetic field due to $g^{*} \sim 0$.

This analysis can also be harnessed to infer some unknown values in the experimental data. To illustrate this point, in Fig. 5(a) the pink star symbol corresponds to InGaAs lens QD having a diameter around $30 \mathrm{~nm}$ and height of 7-8 nm [30]
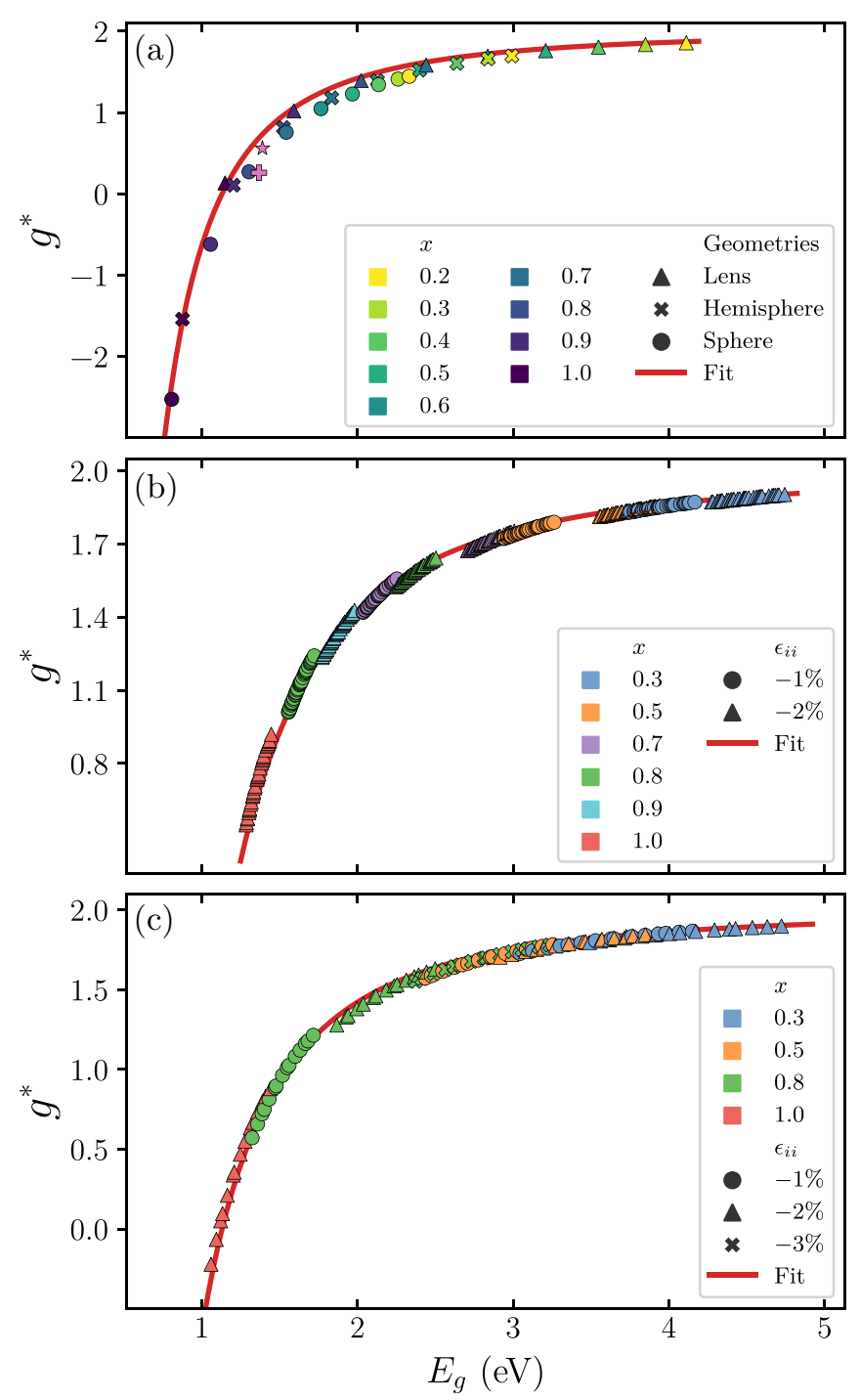

FIG. 5. Universal $g$-factor behavior obtained when the data for (a) different geometries in Fig. 2, (b) $D$ series in Fig. 3, and (c) $h$ series at $D=35 \mathrm{~nm}$ in Fig. 4 are redrawn with respect to $E_{g}$. See the text for two other literature data points, included in (a) as star [30] and cross [35] symbols in pink. All fitted curves obey Eq. (13).

and the pink cross to another QD measured by photocurrent spectroscopy [35], for both of which only the magnitude of $g^{*}$ could be reported. Using Eq. (13) we can resolve either one to be positive. From a more general angle, this universality warrants a recipe by merely tuning the gap energy through any means for the pursuit of $g$-factor engineering [28,79].

\section{CONCLUSIONS}

Using an empirical pseudopotential atomistic electronic structure theory, $g$ tensors of a large number of embedded InGaAs QDs with different shape, size, indium fraction, and strain combinations are computed. This analysis provides the general traits of $g$-factor variation. For specific applications, when taken into account in their growth control or post-selection, it can be beneficial for achieving $g$-near-zero InGaAs QDs or direct ESR-based quantum logic operations. Our study also validates a recent report based on tight-binding 
electronic structure for compound QDs that the $g$ factor acquires a universal behavior with respect to the gap energy of the QD regardless of its structural details [50]. We additionally exhibit that this applies to alloy InGaAs QDs of various shapes and finite confinement allowing for penetration to the matrix. It remains to be examined whether these conclusions will be affected by an inhomogeneous atomic scale strain distribution within the QD.

\section{ACKNOWLEDGMENTS}

This work was funded by Türkiye Bilimsel ve Teknolojik Araştirma Kurumu (TUBITAK) under Project No. 116F075. The numerical calculations reported in this paper were partially performed at TÜBITTAK ULAKBIM, High Performance and Grid Computing Center (TRUBA resources).
[1] Y. Yafet, g factors and spin-lattice relaxation of conduction electrons, in Solid State Physics, Vol. 14 (Elsevier, New York, London, 1963), pp. 1-98.

[2] J. Callaway, Quantum Theory of the Solid State (Academic Press, New York, 2013).

[3] M. A. T. Sandoval, J. E. Leon Padilla, A. Ferreira da Silva, E. A. de Andrada e Silva, and G. C. La Rocca, Mesoscopic g-factor renormalization for electrons in iii-v interacting nanolayers, Phys. Rev. B 98, 075312 (2018).

[4] T. Nakaoka, S. Tarucha, and Y. Arakawa, Electrical tuning of the $g$ factor of single self-assembled quantum dots, Phys. Rev. B 76, 041301(R) (2007).

[5] Y. K. Kato and D. D. Awschalom, Electrical manipulation of spins in nonmagnetic semiconductors, J. Phys. Soc. Jpn. 77, 031006 (2008).

[6] F. Klotz, V. Jovanov, J. Kierig, E. C. Clark, D. Rudolph, D. Heiss, M. Bichler, G. Abstreiter, M. S. Brandt, and J. J. Finley, Observation of an electrically tunable exciton $\mathrm{g}$ factor in ingaas/gaas quantum dots, Appl. Phys. Lett. 96, 053113 (2010).

[7] J. Pingenot, C. E. Pryor, and M. E. Flatté, Electric-field manipulation of the landé $g$ tensor of a hole in an in ${ }_{0.5} \mathrm{ga}_{0.5}$ as/gaas self-assembled quantum dot, Phys. Rev. B 84, 195403 (2011).

[8] M. W. Taylor, P. Spencer, E. Clarke, E. Harbord, and R. Murray, Tuning excitong-factors in InAs/GaAs quantum dots, J. Phys. D: Appl. Phys. 46, 505105 (2013).

[9] C. Santori, D. Fattal, J. Vučković, G. S. Solomon, and Y. Yamamoto, Indistinguishable photons from a single-photon device, Nature (London) 419, 594 (2002).

[10] Y.-M. He, Y. He, Y.-J. Wei, D. Wu, M. Atatüre, C. Schneider, S. Höfling, M. Kamp, C.-Y. Lu, and J.-W. Pan, On-demand semiconductor single-photon source with near-unity indistinguishability, Nat. Nanotechnol. 8, 213 (2013).

[11] A. N. Vamivakas, Y. Zhao, C.-Y. Lu, and M. Atatüre, Spinresolved quantum-dot resonance fluorescence, Nat. Phys. 5, 198 (2009)

[12] S. T. Y1lmaz, P. Fallahi, and A. Imamoğlu, Quantum-dotSpin Single-Photon Interface, Phys. Rev. Lett. 105, 033601 (2010).

[13] R. M. Stevenson, A. J. Hudson, A. J. Bennett, R. J. Young, C. A. Nicoll, D. A. Ritchie, and A. J. Shields, Evolution of Entanglement between Distinguishable Light States, Phys. Rev. Lett. 101, 170501 (2008).

[14] W. B. Gao, P. Fallahi, E. Togan, J. Miguel-Sánchez, and A. Imamoglu, Observation of entanglement between a quantum dot spin and a single photon, Nature (London) 491, 426 (2012).

[15] C. H. Schulte, J. Hansom, A. E. Jones, C. Matthiesen, C. Le Gall, and M. Atatüre, Quadrature squeezed photons from a twolevel system, Nature (London) 525, 222 (2015).
[16] M. Kroner, K. M. Weiss, B. Biedermann, S. Seidl, S. Manus, A. W. Holleitner, A. Badolato, P. M. Petroff, B. D. Gerardot, R. J. Warburton, and K. Karrai, Optical Detection of SingleElectron Spin Resonance in a Quantum dot, Phys. Rev. Lett. 100, 156803 (2008).

[17] F. H. Koppens, C. Buizert, K.-J. Tielrooij, I. T. Vink, K. C. Nowack, T. Meunier, L. Kouwenhoven, and L. Vandersypen, Driven coherent oscillations of a single electron spin in a quantum dot, Nature (London) 442, 766 (2006).

[18] B. Urbaszek, X. Marie, T. Amand, O. Krebs, P. Voisin, P. Maletinsky, A. Högele, and A. Imamoglu, Nuclear spin physics in quantum dots: An optical investigation, Rev. Mod. Phys. 85, 79 (2013).

[19] S. R. Hartmann and E. L. Hahn, Nuclear double resonance in the rotating frame, Phys. Rev. 128, 2042 (1962).

[20] P. London, J. Scheuer, J.-M. Cai, I. Schwarz, A. Retzker, M. B. Plenio, M. Katagiri, T. Teraji, S. Koizumi, J. Isoya, R. Fischer, L. P. McGuinness, B. Naydenov, and F. Jelezko, Detecting and Polarizing Nuclear Spins with Double Resonance on a Single Electron Spin, Phys. Rev. Lett. 111, 067601 (2013).

[21] G.-Q. Liu, Q.-Q. Jiang, Y.-C. Chang, D.-Q. Liu, W.-X. Li, C.-Z. Gu, H. C. Po, W.-X. Zhang, N. Zhao, and X.-Y. Pan, Protection of center spin coherence by dynamic nuclear spin polarization in diamond, Nanoscale 6, 10134 (2014).

[22] S. R. Julian and M. R. Norman, Genetics and g-factors, Nat. Phys. 7, 191 (2011).

[23] V. F. Mitrović, M. Horvatić, C. Berthier, S. A. Lyon, and M. Shayegan, Nmr study of large skyrmions in $\mathrm{al}_{0.13} \mathrm{ga}_{0.87}$ As quantum wells, Phys. Rev. B 76, 115335 (2007).

[24] A. Ulhaq, Q. Duan, E. Zallo, F. Ding, O. G. Schmidt, A. I. Tartakovskii, M. S. Skolnick, and E. A. Chekhovich, Vanishing electron $g$ factor and long-lived nuclear spin polarization in weakly strained nanohole-filled gaas/algaas quantum dots, Phys. Rev. B 93, 165306 (2016).

[25] M. Kuwahara, T. Kutsuwa, K. Ono, and H. Kosaka, Single charge detection of an electron created by a photon in a $\mathrm{g}$ factor engineered quantum dot, Appl. Phys. Lett. 96, 163107 (2010).

[26] M. Bayer, A. Kuther, A. Forchel, A. Gorbunov, V. B. Timofeev, F. Schäfer, J. P. Reithmaier, T. L. Reinecke, and S. N. Walck, Electron and Hole $g$ Factors and Exchange Interaction from Studies of the Exciton Fine Structure in $\mathrm{in}_{0.60} \mathrm{ga}_{0.40}$ As Quantum dots, Phys. Rev. Lett. 82, 1748 (1999).

[27] T. Nakaoka, T. Saito, J. Tatebayashi, and Y. Arakawa, Size, shape, and strain dependence of the $g$ factor in self-assembled in(ga)as quantum dots, Phys. Rev. B 70, 235337 (2004).

[28] T. Nakaoka, T. Saito, J. Tatebayashi, S. Hirose, T. Usuki, N. Yokoyama, and Y. Arakawa, Tuning of $g$-factor in 
self-assembled in(ga)as quantum dots through strain engineering, Phys. Rev. B 71, 205301 (2005).

[29] T. P. M. Alegre, F. G. G. Hernández, A. L. C. Pereira, and G. Medeiros-Ribeiro, Landé $g$ Tensor in Semiconductor Nanostructures, Phys. Rev. Lett. 97, 236402 (2006).

[30] A. Schwan, B.-M. Meiners, A. B. Henriques, A. D. B. Maia, A. A. Quivy, S. Spatzek, S. Varwig, D. R. Yakovlev, and M. Bayer, Dispersion of electron g-factor with optical transition energy in (in,ga)as/gaas self-assembled quantum dots, Appl. Phys. Lett. 98, 233102 (2011).

[31] A. Schwan, B.-M. Meiners, A. Greilich, D. R. Yakovlev, M. Bayer, A. D. B. Maia, A. A. Quivy, and A. B. Henriques, Anisotropy of electron and hole g-factors in (in,ga)as quantum dots, Appl. Phys. Lett. 99, 221914 (2011).

[32] L. Sapienza, R. Al-Khuzheyri, A. Dada, A. Griffiths, E. Clarke, and B. D. Gerardot, Magneto-optical spectroscopy of single charge-tunable inas/gaas quantum dots emitting at telecom wavelengths, Phys. Rev. B 93, 155301 (2016).

[33] H. M. G. A. Tholen, J. S. Wildmann, A. Rastelli, R. Trotta, C. E. Pryor, E. Zallo, O. G. Schmidt, P. M. Koenraad, and A. Y. Silov, Strain-induced $g$-factor tuning in single ingaas/gaas quantum dots, Phys. Rev. B 94, 245301 (2016).

[34] H. M. G. A. Tholen, J. S. Wildmann, A. Rastelli, R. Trotta, C. E. Pryor, E. Zallo, O. G. Schmidt, P. M. Koenraad, and A. Y. Silov, Active tuning of the $g$-tensor in ingaas/gaas quantum dots via strain, Phys. Rev. B 99, 195305 (2019).

[35] S. Wu, K. Peng, X. Xie, J. Yang, S. Xiao, F. Song, J. Dang, S. Sun, L. Yang, Y. Wang, S. Shi, J. He, Z. Zuo, and X. Xu, Electron and Hole $g$ Tensors of Neutral and Charged Excitons in Single Quantum Dots by High-Resolution Photocurrent Spectroscopy, Phys. Rev. Appl. 14, 014049 (2020).

[36] A. A. Kiselev, E. L. Ivchenko, and U. Rössler, Electron g factor in one- and zero-dimensional semiconductor nanostructures, Phys. Rev. B 58, 16353 (1998).

[37] C. E. Pryor and M. E. Flatté, Landé $g$ Factors and Orbital Momentum Quenching in Semiconductor Quantum dots, Phys. Rev. Lett. 96, 026804 (2006).

[38] R. Zielke, F. Maier, and D. Loss, Anisotropic $g$ factor in inas self-assembled quantum dots, Phys. Rev. B 89, 115438 (2014).

[39] K. Gawarecki, Spin-orbit coupling and magnetic-field dependence of carrier states in a self-assembled quantum dot, Phys. Rev. B 97, 235408 (2018).

[40] A. Mielnik-Pyszczorski, K. Gawarecki, and P. Machnikowski, Limited accuracy of conduction band effective mass equations for semiconductor quantum dots, Sci. Rep. 8, 2873 (2018).

[41] C. J. Delerue and M. Lannoo, Nanostructures: Theory and Modeling (Springer Science \& Business Media, Berlin, New York, 2013).

[42] M. Usman, Atomistic theoretical study of electronic and polarization properties of single and vertically stacked elliptical inas quantum dots, Phys. Rev. B 86, 155444 (2012).

[43] C. E. Pryor and M.-E. Pistol, Atomistic k.p theory, J. Appl. Phys. 118, 225702 (2015).

[44] A. Mittelstädt, L. A. T. Greif, S. T. Jagsch, and A. Schliwa, Efficient electronic structure calculations for extended systems of coupled quantum dots using a linear combination of quantum dot orbitals method, arXiv:1912.03983.

[45] V. Mlinar and A. Zunger, Spectral barcoding of quantum dots: Deciphering structural motifs from the excitonic spectra, Phys. Rev. B 80, 035328 (2009).
[46] V. Mlinar, M. Bozkurt, J. M. Ulloa, M. Ediger, G. Bester, A. Badolato, P. M. Koenraad, R. J. Warburton, and A. Zunger, Structure of quantum dots as seen by excitonic spectroscopy versus structural characterization: Using theory to close the loop, Phys. Rev. B 80, 165425 (2009).

[47] A. D. Giddings, J. G. Keizer, M. Hara, G. J. Hamhuis, H. Yuasa, H. Fukuzawa, and P. M. Koenraad, Composition profiling of inas quantum dots and wetting layers by atom probe tomography and cross-sectional scanning tunneling microscopy, Phys. Rev. B 83, 205308 (2011).

[48] J. G. Keizer, M. Jo, T. Mano, T. Noda, K. Sakoda, and P. M. Koenraad, Structural atomic-scale analysis of gaas/algaas quantum wires and quantum dots grown by droplet epitaxy on a (311)a substrate, Appl. Phys. Lett. 98, 193112 (2011).

[49] L. M. Roth, B. Lax, and S. Zwerdling, Theory of optical magneto-absorption effects in semiconductors, Phys. Rev. 114, 90 (1959).

[50] A. Tadjine, Y.-M. Niquet, and C. Delerue, Universal behavior of electron $g$-factors in semiconductor nanostructures, Phys. Rev. B 95, 235437 (2017).

[51] F. Guffarth, R. Heitz, A. Schliwa, O. Stier, N. N. Ledentsov, A. R. Kovsh, V. M. Ustinov, and D. Bimberg, Strain engineering of self-organized inas quantum dots, Phys. Rev. B 64, 085305 (2001).

[52] M. Usman, V. Tasco, M. T. Todaro, M. De Giorgi, E. P. O'Reilly, G. Klimeck, and A. Passaseo, The polarization response in inas quantum dots: Theoretical correlation between composition and electronic properties, Nanotechnology 23, 165202 (2012).

[53] C. Bulutay, Quadrupolar spectra of nuclear spins in strained in $_{x} \mathrm{ga}_{1-x}$ as quantum dots, Phys. Rev. B 85, 115313 (2012).

[54] S. Golovynskyi, O. I. Datsenko, L. Seravalli, G. Trevisi, P. Frigeri, B. Li, D. Lin, and J. Qu, Inas/ingaas quantum dots confined by inalas barriers for enhanced room temperature light emission: Photoelectric properties and deep levels, Microelectron. Eng. 238, 111514 (2021).

[55] H. Von Wenckstern, D. Splith, M. Purfürst, Z. Zhang, C. Kranert, S. Müller, M. Lorenz, and M. Grundmann, Structural and optical properties of (in, ga) 203 thin films and characteristics of schottky contacts thereon, Semicond. Sci. Technol. 30, 024005 (2015).

[56] L.-W. Wang and A. Zunger, Linear combination of bulk bands method for large-scale electronic structure calculations on strained nanostructures, Phys. Rev. B 59, 15806 (1999).

[57] C. Bulutay, Interband, intraband, and excited-state direct photon absorption of silicon and germanium nanocrystals embedded in a wide band-gap lattice, Phys. Rev. B 76, 205321 (2007).

[58] H. Yildırım and C. Bulutay, Bound-state third-order optical nonlinearities of germanium nanocrystals embedded in a silica host matrix, Phys. Rev. B 78, 115307 (2008).

[59] C. Bulutay, M. Kulakci, and R. Turan, Stark effect, polarizability, and electroabsorption in silicon nanocrystals, Phys. Rev. B 81, 125333 (2010).

[60] D. Gunceler and C. Bulutay, dc-switchable and singlenanocrystal-addressable coherent population transfer, Appl Phys. Lett. 97, 241909 (2010).

[61] Ü. Keleş, B. Liedke, K.-H. Heinig, and C. Bulutay, Networks of silicon nanowires: A large-scale atomistic electronic structure analysis, Appl. Phys. Lett. 103, 203103 (2013). 
[62] Ü. Keleş, A. Çakan, and C. Bulutay, Disorder-free localization around the conduction band edge of crossing and kinked silicon nanowires, J. Appl. Phys. 117, 064308 (2015).

[63] A. Bechtold, D. Rauch, F. Li, T. Simmet, P.-L. Ardelt, A. Regler, K. Müller, N. A. Sinitsyn, and J. J. Finley, Three-stage decoherence dynamics of an electron spin qubit in an optically active quantum dot, Nat. Phys. 11, 1005 (2015).

[64] G. Bester, Electronic excitations in nanostructures: An empirical pseudopotential based approach, J. Phys.: Condens. Matter 21, 023202 (2008).

[65] M. S. Hybertsen and S. G. Louie, Spin-orbit splitting in semiconductors and insulators from the ab initio pseudopotential, Phys. Rev. B 34, 2920 (1986).

[66] A. J. Williamson, L. W. Wang, and A. Zunger, Theoretical interpretation of the experimental electronic structure of lens-shaped self-assembled inas/gaas quantum dots, Phys. Rev. B 62, 12963 (2000).

[67] L. M. Roth, $g$ factor and donor spin-lattice relaxation for electrons in germanium and silicon, Phys. Rev. 118, 1534 (1960).

[68] A. Çakan, C. Sevik, and C. Bulutay, Strained band edge characteristics from hybrid density functional theory and empirical pseudopotentials: GaAs, GaSb, InAs and InSb, J. Phys. D: Appl. Phys. 49, 085104 (2016).

[69] M. Frigo, A fast fourier transform compiler, in Proceedings of the ACM SIGPLAN 1999 Conference on Programming Language Design and Implementation (Association for Computing Machinery, New York, 1999), pp. 169-180.

[70] C. G. Van de Walle, Band lineups and deformation potentials in the model-solid theory, Phys. Rev. B 39, 1871 (1989).
[71] C. Pryor, Eight-band calculations of strained inas/gaas quantum dots compared with one-, four-, and six-band approximations, Phys. Rev. B 57, 7190 (1998).

[72] S. Ghosh, B. Kochman, J. Singh, and P. Bhattacharya, Conduction band offset in inas/gaas self-organized quantum dots measured by deep level transient spectroscopy, Appl. Phys. Lett. 76, 2571 (2000).

[73] M. Usman, H. Ryu, I. Woo, D. S. Ebert, and G. Klimeck, Moving toward nano-tcad through multimillion-atom quantumdot simulations matching experimental data, IEEE Trans. Nanotechnol. 8, 330 (2009).

[74] D. Bimberg, M. Grundmann, and N. N. Ledentsov, Quantum Dot Heterostructures (John Wiley \& Sons, Chichester, New York, 1999).

[75] G. Klimeck, S. S. Ahmed, H. Bae, N. Kharche, S. Clark, B. Haley, S. Lee, M. Naumov, H. Ryu, F. Saied et al., Atomistic simulation of realistically sized nanodevices using nemo 3dpart i: Models and benchmarks, IEEE Trans. Electron Devices 54, 2079 (2007).

[76] A. H. Barnett, J. Magland, and L. af Klinteberg, A parallel nonuniform fast fourier transform library based on an "exponential of semicircle" kernel, SIAM J. Sci. Comput. 41, C479 (2019).

[77] A. H. Barnett, Aliasing error of the $\exp \left(\beta \sqrt{1-z^{2}}\right)$ kernel in the nonuniform fast fourier transform, arXiv:2001.09405.

[78] Y. Peter and M. Cardona, Fundamentals of Semiconductors: Physics and Materials Properties (Springer, Berlin, Heidelberg, 2010).

[79] G. Medeiros-Ribeiro, E. Ribeiro, and H. Westfahl Jr., g-factor engineering and control in self-assembled quantum dots, Appl. Phys. A 77, 725 (2003). 